\title{
An online module to evaluate the validity of an algorithm to assess the risk for drug-induced QTC prolongation in the psychiatric population
}

\author{
Iman Qubaiah ${ }^{1}$, Waad Elamin ${ }^{1}$, Shorouk Akram Homs ${ }^{1}$, Doaa Mahmoud ${ }^{1}$, Enge Tawfik ${ }^{1}$, Monica Zolezzi ${ }^{1}$, Athar Elhakim ${ }^{2}$ \\ ${ }^{1}$ College of Pharmacy, Qatar University, Doha, Qatar \\ ${ }^{2}$ School of Health Sciences, College of North Atlantic Qatar, Doha, Qatar
}

\section{Introduction}

QTC interval prolongation leads to serious complications.

Assessing the risk of QTc interval prolongation can be challenging for clinicians especially in the psychiatric population.

An algorithm was developed to overcome this challenge and assist clinicians when prescribing decisions need to be made.

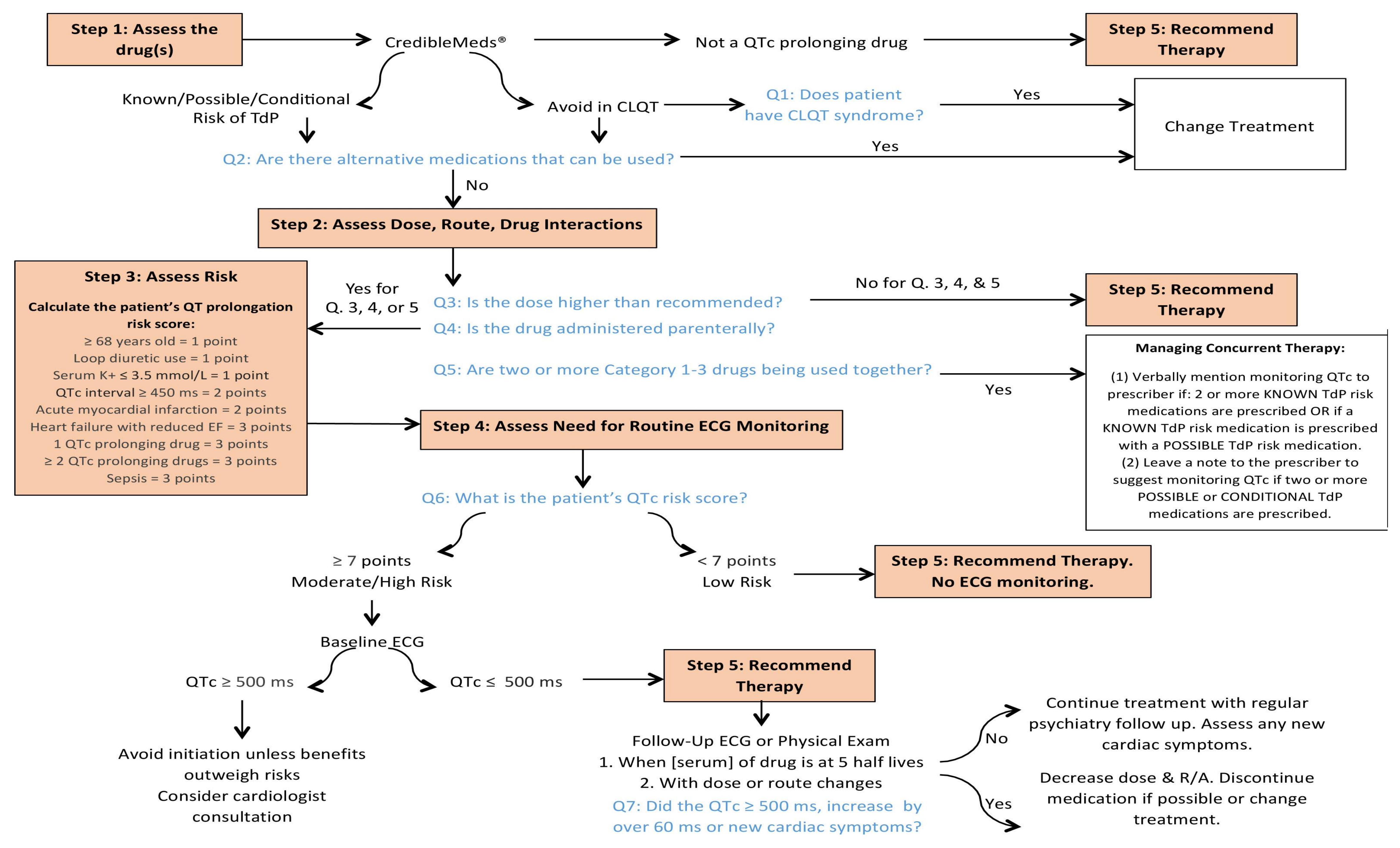

Figure 1. Stepped-based algorithm for assessing QTc prolongation risk

\section{Aim}

To develop and pilot test an online educational module to guide users on the stepped-based algorithm developed to assess the risk of drug-induced QTC prolongation in the psychiatric population.
ᄃ

$>$ Articulate $360^{\circledR}$ software was used to develop the online educational module.

$>$ This software consists of several applications that were used to assist in interactive learning.

$>$ Online module included an introduction to the algorithm, case studies and an online survey.

$>$ Online module was pilot tested and distributed to a sample of Faculty members to gather feedback on its format and usability, and then to cardiologists to gather feedback regarding the content validity of the algorithm.

\section{Results}

Initial pilot testing by 3 faculty members indicated that the online module interface was too busy.

$>$ Feedback was utilized to publish another simpler version.

$>$ Pilot testing of the second version was carried out and feedback was gathered from a cardiologist.

$>$ Online educational module provided a thorough explanation of the algorithm steps and rationale.

- Minor issues were raised regarding audio voice overs when transitioning between slides.

$>$ Feedback gathered was incorporated and utilized to produce a final version of the educational module.

> Additional feedback was received regarding the algorithm itself after sending a cross-sectional, anonymous, self-administered survey to 20 cardiologist. 12 responses were received.

> Preliminary survey results have been positive based on the open-ended survey responses (algorithm is easy to use, systematic and likely helpful if implemented), and is thought to be appropriate, safe and reliable based on the closed-ended survey responses.

D Small portion stated that it is complicated and cumbersome.

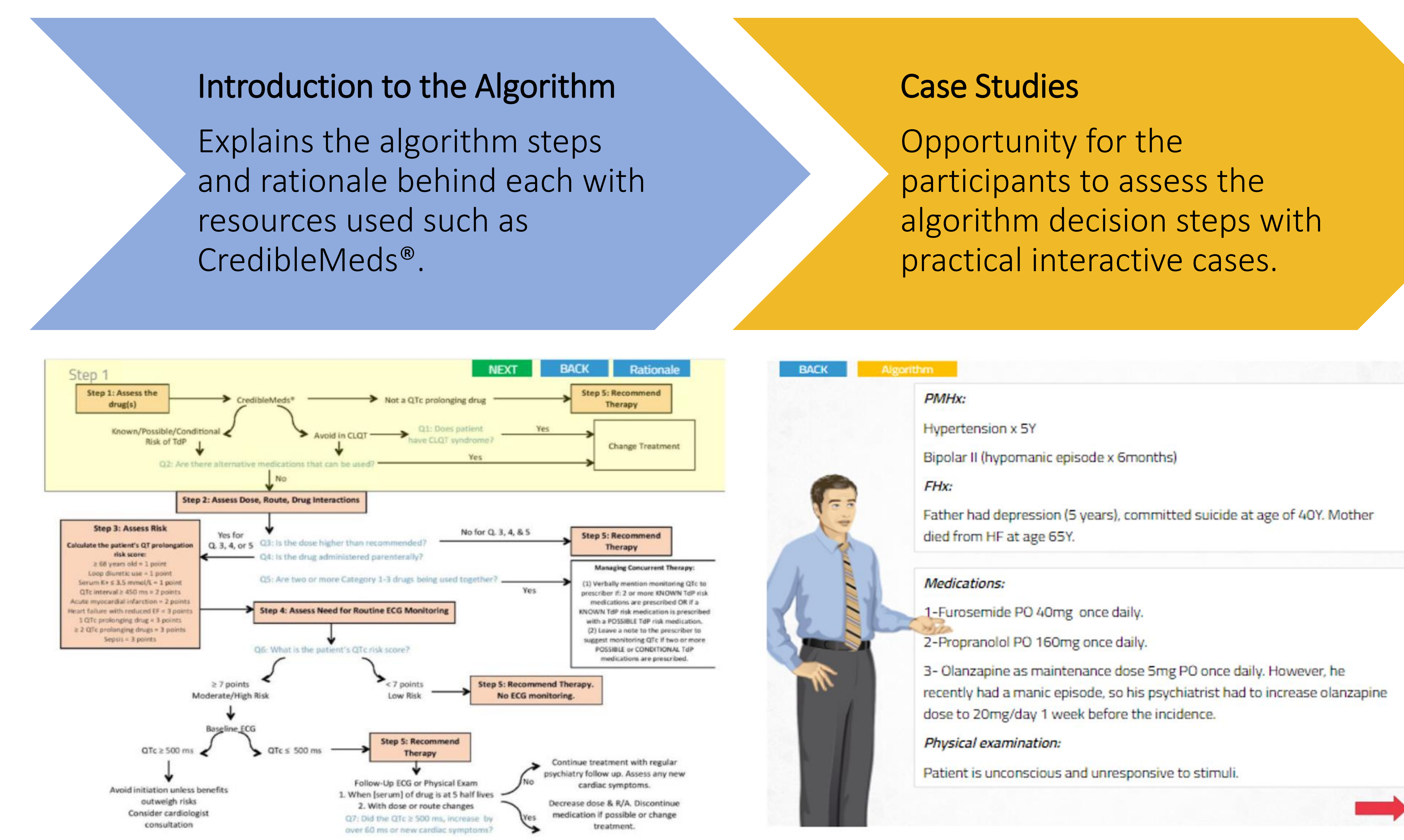

Figure 2. Overview of Algorithm Steps

Figure 3. Sample Case Study

\section{Conclusion}

- The online educational module was finalized based on the pilot-testing feedback and used to orient research participants to the QTc prolongation algorithm prior to distributing an online survey to assess algorithm's content validity.

$>$ Feedback received on the algorithm has been promising thus far, as most cardiologists have supported the use of the algorithm. The negative feedback will be further investigated and incorporated to make the algorithm easier to use and follow.

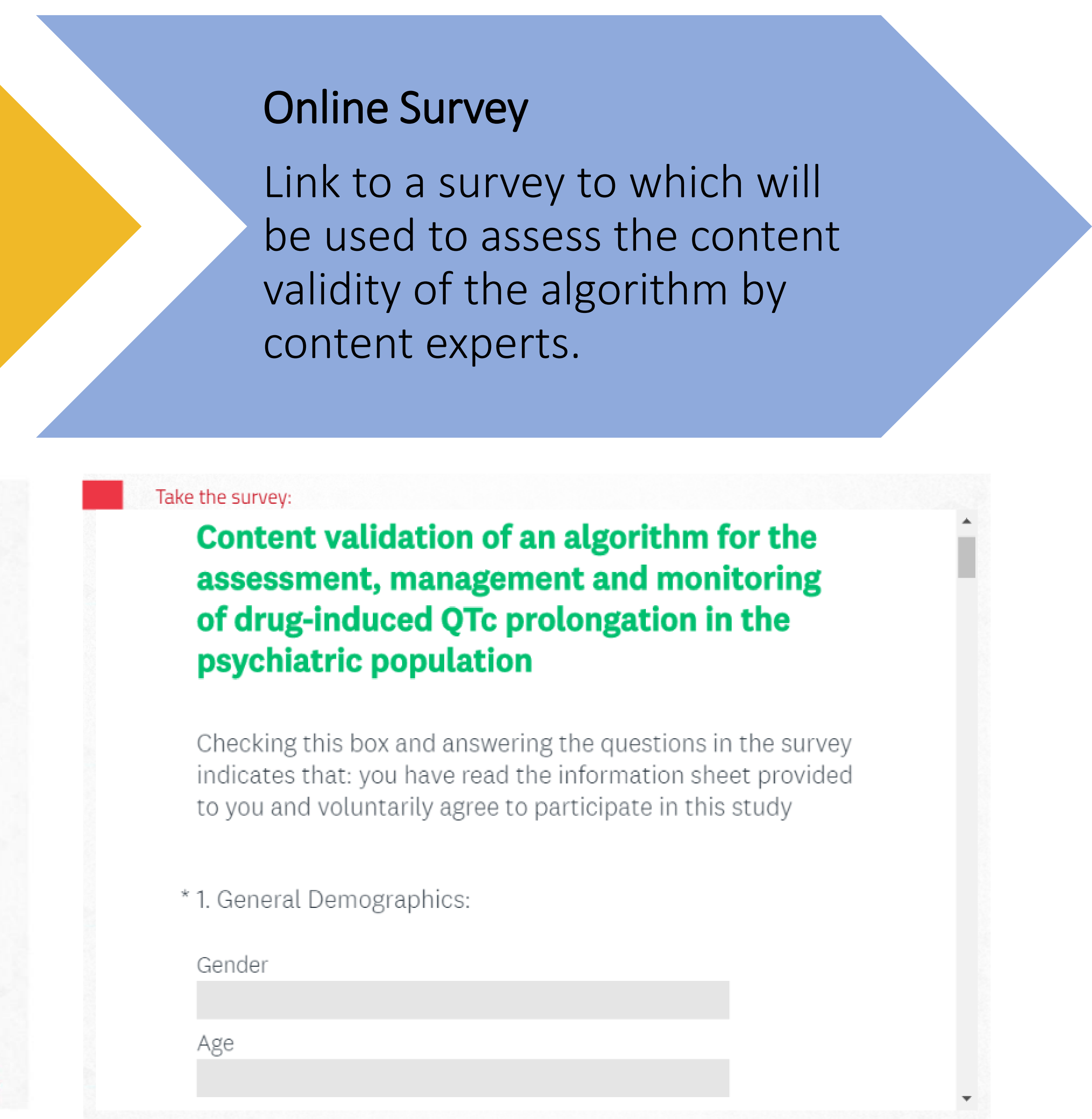

Figure 4. Online Survey

\section{Acknowledgements}

$>$ Presentation was made possible by a UREP grant (UREP24041-3-016) from the Qatar National Research Fund.

$>$ The statements made herein are solely the responsibility of the authors. 